\title{
TOPOLOGICAL MINIMAX THEOREMS
}

\author{
MICHAEL A. GERAGHTY AND BOR-LUH LIN ${ }^{1}$
}

ABStraCt. Mimimax theorems are given using only topological conditions.

Most mimimax theorems involve linear structure (see references in [4 and 6]). Minimax theorems without any linear structure have been obtained by Fan [4], Terkelsen [6] and $\mathrm{Wu}[\mathbf{7}]$. In this paper, we shall prove some minimax theorems with only topological conditions. The result of $\mathrm{Wu}$ is a consequence of our theorems.

Let $X$ be a compact (Hausdorff) space and let $U(X)$ be the family of all realvalued upper semicontinuous functions on $X$, i.e., $U(X)=C\left(X, \mathbf{R}^{*}\right)$, where $\mathbf{R}^{*}$ is the set of reals with the topology of upper semicontinuity. We require that the topology on $U(X)$ have the property that the evaluation function $e_{x}: U(X) \rightarrow \mathbf{R}^{*}$, where $e_{x}(f)=f(x)$ for all $f$ in $\mathcal{U}(X)$, be continuous for each $x$ in $X$. Since the range space $\mathbf{R}^{*}$ is again the set of reals with the topology of upper semicontinuity, $e_{x}$ is an upper semicontinuous function from $\mathcal{U}(X)$ to the reals $\mathbf{R}$ with the usual topology. In particular, this will hold for "conjoining" topologies in the sense of Dugundji [3, p. 274]. For additional material on the convergence of upper semicontinuous functions and related topologies, see [2 and 5] and references cited therein.

DEFINITION. A subset $\mathcal{F}$ of $\mathcal{U}(X)$ is said to be submaximum if for any $f, g$ in $\mathcal{F}$ there exists a continuous function $S:[0,1] \rightarrow \mathcal{F}$ such that $S(0)=f, S(1)=g$ and for any $b \in[a, c] \subset[0,1], S(b)(x) \leq \max \{S(a)(x), S(c)(x)\}$ for all $x \in X$.

Observe that the last condition is easily seen to be equivalent to the condition that $L_{x}^{\alpha}=\{t: t \in[0,1], S(t)(x)<\alpha\}$ is either connected or empty for all $x$ in $X$ and for all $\alpha \in \mathbf{R}$. This will be satisfied if for all $x$ in $X$ the evaluation mapping from $\mathcal{F}$ to $\mathbf{R}$ defined by $f \mapsto f(x)$ is quasiconvex $[\mathbf{1}]$ on the set $\mathcal{F}$. Since we assume that $e_{x}$ is continuous on $U(X), L_{x}^{\alpha}$ is open in $[0,1]$. It is clear that every convex subset in $\mathcal{U}(X)$ is submaximum.

LEMMA 1. Let $X$ be a compact space and let $\mathcal{F}$ be a submaximum set in $U(X)$. Suppose that for some $\alpha \in \mathbf{R}$, the set $A_{f}^{\alpha}=\{x: x \in X, f(x) \geq \alpha\}$ is either empty or connected for all $f$ in $\mathcal{F}$. Then for any $g_{0}, g_{1}$ in $\mathcal{F}$ such that $\min \left\{g_{0}(x), g_{1}(x)\right\}<\alpha$ for all $x$ in $X$, there exists an element $g$ in $\mathcal{F}$ such that $g(x)<\alpha$ for all $x$ in $X$.

PROOF. Let $S:[0,1] \rightarrow \mathcal{F}$ be a continuous mapping such that $S(0)=g_{0}, S(1)=$ $g_{1}$ and $S(b)(x) \leq \max \{S(a)(x), S(c)(x)\}$ for all $x$ in $X$ and for any $b \in[a, c] \subset[0,1]$. For each $x$ in $X$, let $L_{x}=\{t: S(t)(x)<\alpha\}$ and $A_{x}=\{t: S(t)(x) \geq \alpha\}$. As remarked above, $L_{x}$ is an open interval.

Received by the editors April 8, 1983 and, in revised form, August 23, 1983.

1980 Mathematics Subject Classification. Primary 49A40; Secondary 26A15.

Key words and phrases. Minimax, upper semicontinuity, submaximum.

${ }^{1}$ Both authors were partially supported by Developmental Assignments from The University of Iowa.

(C) 1984 American Mathematical Society $0002-9939 / 84 \$ 1.00+\$ .25$ per page 
Now, for each $f \in \mathcal{F}$, let $L_{f}=\{x: x \in X, f(x)<\alpha\}$ and let $A_{f}=\{x: x \in$ $X, f(x) \geq \alpha\}$. Since $\min \left\{g_{0}(x), g_{1}(x)\right\}<\alpha, X=L_{g_{0}} \cup L_{g_{1}}$. Observe that if $X=L_{g_{0}}$ (respectively, $X=L_{g_{1}}$ ), then we may choose $g=g_{0}$ (respectively, $g=g_{1}$ ). We need only consider the case when $A_{g_{0}}$ and $A_{g_{1}}$ are nonempty compact subsets of $X$. Note that if $x \in A_{g_{0}}$ then $x \in L_{g_{1}}$ and so $1 \in L_{x}$. Similarly, $x \in A_{g_{1}}$ implies that $0 \in L_{x}$. Thus we may write, for each $x$ in $X$,

$$
L_{x}= \begin{cases}\left(M_{x}, 1\right] & \text { if } x \in A_{g_{0}}, \\ {\left[0, m_{x}\right)} & \text { if } x \in A_{g_{1}}, \\ {[0,1]} & \text { if } x \in L_{g_{0}} \cap L_{g_{1}} .\end{cases}
$$

Let $m=\inf _{x \in A_{g_{1}}} m_{x}$ and $M=\sup _{x \in A_{g_{0}}} M_{x}$. Choose $x_{n}$ in $A_{g_{1}}$ such that $\left\{m_{x_{n}}\right\}$ decreases to $m$, and moreover, such that $\left\{x_{n}\right\}$ converges to some element $x_{0}$ in $A_{g_{1}} \subset L_{g_{0}}$. Fix $k$. For any $n>k$, we have $m_{x_{n}} \leq m_{x_{k}}$. Hence $m_{x_{k}} \in A_{x_{n}}$. It follows that $x_{n} \in A_{S\left(m_{x_{k}}\right)}$, a closed set in $X$ by the upper semicontinuity of $S\left(m_{x_{k}}\right)$, for all $n>k$. Thus $x_{0} \in A_{S\left(m_{x_{k}}\right)}$ for all $k=1,2, \ldots$ So $m_{x_{k}} \in A_{x_{0}}$ for all $k$. This implies that $m \in A_{x_{0}}$. Since $m=\inf _{x \in A_{g_{1}}} m_{x}$, we conclude that $A_{x_{0}}=[m, 1]$ and $L_{x_{0}}=[0, m)$. Similarly, we obtain an element $x_{1}$ in $A_{g_{0}}$ such that $A_{x_{1}}=[0, M]$ and $L_{x_{1}}=(M, 1]$. Now $\bigcap_{x \in X} L_{x}=[0, m) \cap(M, 1]$. If $M<m$, choose $M<t<m$ and set $g=S(t)$. Since $t \in L_{x}$ for all $x \in X$, we have $g(x)=S(t)(x)<\alpha$ for all $x$ in $X$. If $m \leq M$, choose $m \leq t \leq M$. Consider the connected set $A_{S(t)}$. We have $L_{g_{0}}$ and $L_{g_{1}}$ open sets, $X=L_{g_{0}} \cup L_{g_{1}} \supset A_{S(t)}, x_{0} \in L_{g_{0}} \cap A_{S(t)}$ and $x_{1} \in L_{g_{1}} \cap A_{S(t)}$. So from the assumption that $A_{S(t)}$ is connected there must exist some $x \in L_{g_{0}} \cap L_{g_{1}} \cap A_{S(t)}$. This implies that $L_{x}=[0,1]$ and we must have $x \in L_{S(t)}$. This contradicts $x \in A_{S(t)}$. Q.E.D.

THEOREM 2. Let $X$ be a compact space and let $\mathcal{F}$ be a submaximum set in $\mathcal{U}(X)$. If for any $f_{1}, f_{2}, \ldots, f_{n}$ in $\mathcal{F}$ and any $\alpha$ in $\mathbf{R}$, the set $\left\{x: x \in X, f_{i}(x) \geq\right.$ $\alpha, i=1,2, \ldots, n\}$ is either connected or empty, then

$$
\sup _{X} \inf _{\mathcal{F}} f(x)=\inf _{\mathcal{F}} \sup _{X} f(x) .
$$

PROOF. It suffices to show that for any $\alpha \in \mathbf{R}$, if $\sup _{X} \inf _{\mathcal{F}} f(x)<\alpha$ then $\inf _{\mathcal{F}} \sup _{X} f(x)<\alpha$. By compactness of $X$, there exist $f_{1}, f_{2}, \ldots, f_{n}$ in $\mathcal{F}$ such that $\sup _{X} \inf \left\{f_{1}(x), \ldots, f_{n}(x)\right\}<\alpha$. It remains to find $g$ in $₹$ such that $g(x)<\alpha$ for all $x$ in $X$. This is done by induction on $n$. For $n=1$, let $g=f_{1}$. Given $\sup _{X} \inf \left\{f_{1}(x), \ldots, f_{n}(x)\right\}<\alpha$, let $X_{n}=A_{f_{n}}^{\alpha}=\left\{x: x \in X, f_{n}(x) \geq \alpha\right\}$. Then $X_{n}$ is compact. Let $\mathcal{F}_{n}=\left\{\left.f\right|_{X_{n}}: f \in \mathcal{F}\right\}$. Then $\mathcal{F}_{n}$ is submaximum in $\mathcal{U}\left(X_{n}\right)$. Apply the induction hypothesis to $\left(X_{n}, g_{1}, \ldots, g_{n-1}\right)$, where $g_{i}=\left.f_{i}\right|_{X_{n}}, i=1,2, \ldots, n-1$. Then there exists $g_{n} \in \mathcal{F}_{n}$ such that $g_{n}(x)<\alpha$ for all $x \in X_{n}$. Let $f \in \mathcal{F}$ be such that $\left.f\right|_{X_{n}}=g_{n}$. Then $\sup _{X} \min \left\{f(x), f_{n}(x)\right\}<\alpha$. By Lemma 1 , there exists $g \in \mathcal{F}$ such that $g(x)<\alpha$ for all $x$ in $X$. This completes the proof of the theorem. Q.E.D.

COROLLARY 3. Let $X$ be a compact convex set and let $₹$ be a submaximum set in $\mathcal{U}(X)$. If $A_{f}^{\alpha}=\{x: x \in X, f(x) \geq \alpha\}$ is convex for all $f$ in $\mathcal{F}$ and for all $\alpha$ in $\mathbf{R}$ then $\sup _{X} \inf _{\mathcal{F}} f(x)=\inf _{\mathcal{F}} \sup _{X} f(x)$.

COROLlaRY 4. Let $X$ be a compact space and let $₹$ be a convex set in $U(X)$. If for any $f_{1}, \ldots, f_{n}$ in $\mathcal{F}$ and any $\alpha$ in $\mathbf{R}$, the set $\left\{x: x \in X, f_{i}(x) \geq \alpha, i=1,2, \ldots, n\right\}$ is either connected or empty then $\sup _{X} \inf _{\mathcal{F}} f(x)=\inf _{\mathcal{F}} \sup _{X} f(x)$. 
LeMmA 5. Let $X$ be a compact space. For any $f_{1}, \ldots, f_{n}$ in $\mathcal{U}(X)$, the sets $\left\{x: x \in X, f_{i}(x) \geq \alpha, i=1,2, \ldots, n\right\}$ are connected or empty for all $\alpha$ in $\mathbf{R}$ if and only if the sets $\left\{x: x \in X, f_{i}(x)>\alpha, i=1,2, \ldots, n\right\}$ are either connected or empty for all $\alpha$ in $\mathbf{R}$.

Proof. For $f \in \mathcal{U}(X)$ and $\alpha \in \mathbf{R}$, let $U_{f}^{\alpha}=\{x: x \in X, f(x)>\alpha\}$.

Assume that $\bigcap_{i=1}^{n} U_{f_{i}}^{\alpha}$ is either connected or empty for all $\alpha \in \mathbf{R}$. Fix $\alpha \in \mathbf{R}$. Let $\left\{\beta_{j}\right\}$ be a sequence increasing to $\alpha$. Then

$$
\bigcap_{i=1}^{n} A_{f_{i}}^{\alpha}=\bigcap_{i=1}^{n} \bigcap_{j=1}^{\infty} U_{f_{i}}^{\beta_{j}} \subset \bigcap_{j=1}^{\infty} \overline{\bigcap_{i=1}^{n} U_{f_{i}}^{\beta_{j}}} \subset \bigcap_{j=1}^{\infty} \bigcap_{i=1}^{n} A_{f_{i}}^{\beta_{j}}=\bigcap_{i=1}^{n} A_{f_{i}}^{\alpha} .
$$

Thus $\bigcap_{i=1} A_{f_{i}}^{\alpha}=\bigcap_{j=1}^{\infty} \overline{\bigcap_{i=1}^{n} U_{f_{i}}^{\beta_{j}}}$. Since $\bigcap_{i=1}^{n} U_{f_{i}}^{\beta j}, j=1,2, \ldots$, is a nested sequence of compact connected sets or empty sets, the intersection, $\bigcap_{j=1}^{\infty} \overline{\bigcap_{i=1}^{n} U_{f_{i}}^{\beta_{j}}}$ is either connected or empty. This shows that $\bigcap_{i=1}^{n} A_{f_{i}}^{\alpha}$ is either connected or empty.

Conversely, assume that $\bigcap_{i=1}^{n} A_{f_{i}}^{\alpha}$ is either connected or empty for all $\alpha$ in $\mathbf{R}$. Fix $\alpha$ in R. Let $\left\{\beta_{j}\right\}$ be a sequence decreasing to $\alpha$. Then

$$
\bigcap_{i=1}^{n} U_{f_{i}}^{\alpha}=\bigcap_{i=1}^{n} \bigcup_{j=1}^{\infty} A_{f_{i}}^{\beta_{j}}=\bigcup_{j=1}^{\infty} \bigcap_{i=1}^{n} A_{f_{i}}^{\beta_{j}}
$$

which is a nested union of either connected or empty sets. Hence $\bigcap_{i=1}^{n} U_{f_{i}}^{\alpha}$ is either connected or empty. Q.E.D.

COROLLARY 6. Let $X$ be a compact space and let $\mp$ be a submaximum set in $\mathcal{U}(X)$. If for any $f_{1}, f_{2}, \ldots, f_{n}$ in $\mathcal{F}$ and any $\alpha$ in $\mathbf{R}$, the sets $\left\{x: x \in X, f_{i}(x)>\right.$ $\alpha, i=1,2, \ldots, n\}$ are either connected or empty, then

$$
\sup _{X} \inf _{\mathcal{F}} f(x)=\inf _{\mathcal{F}} \sup _{X} f(x) \text {. }
$$

COROLlaRY 7 (WU [7]). Let $X$ be a separable compact space and let $Y$ be arcwise connected. If $f: X \times Y \rightarrow \mathbf{R}$ is a mapping such that for any $x \in X, y \in Y$, the mappings $f_{x}(y)=f(x, y)=f_{y}(x)$ are continuous and, moreover, $f$ possesses the two properties:

(1) for any $y_{0}, y_{1}$ in $Y$, there exists a continuous mapping $h:[0,1] \rightarrow Y$ such that $h(0)=y_{0}, h(1)=y_{1}$ and the set $\{t: f(x, h(t)) \geq \alpha\}$ is either connected or empty for all $x$ in $X$ and for all $\alpha$ in $\mathbf{R}$,

(2) for any $y_{1}, \ldots, y_{n}$ in $Y$ and any $\alpha$ in $\mathbf{R}$, the set $\left\{x: x \in X, f\left(x, y_{i}\right)<\lambda, i=\right.$ $1,2, \ldots, n\}$ is either connected or empty,

then $\inf _{X} \sup _{Y} f(x, y)=\sup _{Y} \inf _{X} f(x, y)$.

Let $X$ be a compact space and let $\mathcal{F}$ be a subset in $U(X) . X$ is said to be supminimum relative to $\mathcal{F}$ if for any $x, y$ in $X$ there exists a continuous mapping $S:[0,1] \rightarrow X$ such that $S(0)=x, S(1)=y$ and for any any $0 \leq a \leq b \leq c \leq 1$, $f(S(b)) \geq \min \{f(S(a)), f(S(c))\}$ for all $f$ in $\mathcal{F}$. If $X$ is convex, this will be satisfied if for any $f \in \mathcal{F}, f$ is a quasiconcave function on $X[\mathbf{1}]$. It is clear that if $\mathcal{F}$ is supminimum relative to $\mathcal{F}$, then the set $\left\{x: x \in X, f_{i}(x) \geq \alpha, i=1,2, \ldots, n\right\}$ is either connected or empty for all $f_{1}, \ldots, f_{n}$ in $\mathcal{F}$ and for all $\alpha$ in $\mathbf{R}$. Thus we obtain 
the following

COROLlARY 8. Let $X$ be a compact space and let $₹$ be a submaximum subset in $\mathcal{U}(X)$. If $X$ is supminimum relative to $\mathcal{F}$ then $\sup _{X} \inf _{\mathcal{F}} f(x)=\inf _{\mathcal{F}} \sup _{X} f(x)$.

Finally, let us give a simple example to show that Lemma 1 fails when $₹$ is not submaximum. Let $X=\{1,-1\}$ with the discrete topology. Let $\mathcal{U}(X)$ have the sup norm topology. For each $y \in[-1,0) \cup(0,1]$, define $f_{y}(1)=y$ and $f_{y}(-1)=$ $-y$. Then $\mathcal{F}=\left\{f_{y}: y \in[-1,0) \cup(0,1]\right\}$ is easily seen to be homeomorphic to $[-1,0) \cup(0,1]$. It is trivial that $\mathcal{F}$ is not submaximum, since there is no path in $\mathcal{F}$ joining $f_{1}$ and $f_{-1}$. Clearly the sets $\left\{x: x \in X, f_{y}(x) \geq 0\right\}$ are connected for all $f_{y}$ in $\mathcal{F}$. However, $\sup _{X} \min \left\{f_{1}(x), f_{-1}(x)\right\}<0$ and $\sup _{X} h(x)>0$ for all $h$ in $\mathcal{F}$.

\section{REFERENCES}

1. J. P Aubin, Mathematical methods of game and economic theory, North-Holland, Amsterdam, 1979.

2. S. Dolecki, G. Salinetti and R. J.-B. Wets, Convergence of functions: Equi-semicontinuity, Trans. Amer. Math. Soc. 276 (1983), 409-429.

3. J. Dugundji, Topology, Allyn \& Bacon, Boston, Mass., 1966.

4. Fan Ky, Minimax theorems, Proc. Nat. Acad. Sci. U.S.A. 39 (1953), 42-47.

5. L. McLinden and R. C. Bergstrom, Preservation of convergence of convex sets and functions in finite dimensions, Trans. Amer. Math. Soc. 268 (1981), 127-142.

6. F. Terkelsen, Some minimax theorems, Math. Scand. 31 (1972), 405-413.

7. Wu Wen-tsün, A remark on the fundamental theorem in the theory of games, Science Record (N.S.) 3 (1959), 229-232.

Department of Mathematics, The University of Iowa, Iowa City, Iowa 52242 